\title{
Humic-like acid derived from 1,2-naphthoquinone
}

\author{
Valentina A. Litvin ${ }^{\mathrm{a}^{*}}$, Roger Abi Njoh ${ }^{\mathrm{b}}$ \\ a Department of Chemistry and Nanomaterial Science, Bohdan Khmelnitsky National University, \\ Shevchenko str. 81, Cherkasy, 18031, Ukraine \\ ${ }^{\mathrm{b}}$ Department of Toxicology, Institute of Health Sciences, Near East University, 99138 Nicosia, \\ North Cyprus

\section{litvinvalentina@ukr.ner}

Keywords: humic-like acid, synthetic humic substances, 1,2-naphthoquinone, redox properties, spectroscopy.

A new fast and original method for producing synthetic humic acids by oxidation of 1,2naphthoquinone by oxygen in an alkaline medium has been proposed. The elemental, structuralgroup composition and spectral characteristics of the obtained humic-like substances correspond to those of natural humic substances. The stoichiometry of the reaction was established by the volumetric method. The redox capacity of humic acids increased from 0.43 to $3.68 \mathrm{mmol} \cdot \mathrm{g}^{-1}$ with increasing solution from $\mathrm{pH} 5$ to 11. Humic-like acids featured an ESR singlet signal with a width of 3.8 Gauss and $g$-factor close to the g-factor of the free electron $(g=2.0033)$.

\section{Introduction}

Humic acids (HAs) are a wide class of compounds with different structure, composition and number of molecular fragments [1]. The main natural sources of humic substances are soil, peat, natural waters and coal. Depending on the type of raw material, low-molecular or high-molecular compounds with different contents of aliphatic and aromatic fragments and functional groups may predominate in the composition of HAs [2].

Structural features of HA allow them to participate in various biochemical reactions, form complex compounds, influence photochemical processes, etc [3]. In addition,
HAs can serve as a source of structural fragments of organic macromolecules during biosynthesis that occurs in living organisms. A number of authors noted that HAs exhibit surface-active properties as colloidal systems $[3,4]$. Experimental studies confirm that they are able to exhibit anti-inflammatory, antibacterial, antioxidant, antiviral, membranotropic and other effects $[5,6]$. However the chemical composition of the HA of different sources is characterized by an individual set of functional groups, molecular weight, ratio of aromatic to aliphatic structures, which ultimately determine their biological properties [7]. The main obstacle to the spread 
in medicine of preparations based on humic substances is the difficulty of standardizing them. Therefore, the urgent task is to develop methods for producing synthetic substances that have physicochemical and therapeutic properties similar to natural HA, but differ in reproducible, controlled and standardized properties.

Until now, few models of humic substances have been synthesised. Some of them were obtained using the Maillard reaction [8], which consists of the reduction of amino acids (melanoidin, glycine and melanoid) with sugars (xylose). Jung et. al. [8] proposed to synthesize humic substances by an oxidative reaction using catechin and one amino acid (glycine) or one dipeptide (triglycine).

Known theory of the formation of humic substances is based on the oxidative processes of numerous phenolic molecules that are formed from plant residues under the influence of soil microorganisms [1]. Therefore, several attempts have been made to synthesize humic substances from phenolic precursors by chemical or enzymatic oxidation [9-12].

1,2-naphthoquinone (Figure 1) is a promising precursor in the synthesis of humic acids because its molecule contains aromatic nuclei and a quinoid structure, which is always present in the structure of molecules of natural HAs.

Naphthoquinones are natural aromatic compounds that have been found in several plant families, as well as isolated from fungi, algae and bacteria [13].

Traditionally, naphthoquinones are used as natural dyes. However, the biological activity of these compounds has recently been reported. The pharmacological activity of naphthoquinones is associated with redox and acid-base properties that can be synthetically modified, thereby enhancing their therapeutic properties [14].

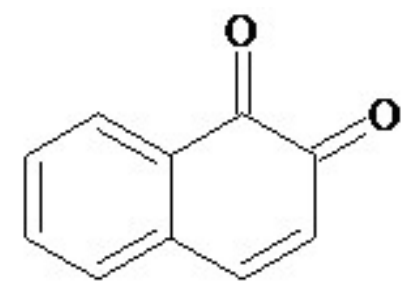

Figure 1. The structural formula of 1,2-naphthoquinone.

In this paper, for the first time, a method for producing humic-like acids by oxidation of reaction of 1,2-naphthoquinone with molecular oxygen is proposed. This method is characterized by simplicity, similarity of the properties of the final product to natural humic substances and reproducibility of the main characteristics.

\section{Experimental part}

\section{Materials and methods}

All chemicals were analytical grade from Merck, Germany and used as received. Solutions were prepared with deionized water (18,2 $\mathrm{M} \Omega / \mathrm{cm}$ resistively) obtained using MilliQ system (Millipore). Molecular oxygen $\left(\mathrm{O}_{2}\right)$ was obtained by electrolysis of $20 \%$ solution of $\mathrm{H}_{2} \mathrm{SO}_{4}$ with platinum anode. 
To determine the elemental composition of HAs, the method of automatic microanalysis was used, which makes it possible to determine the content of three elements. The determination process can be schematically divided into three stages: oxidative pyrolysis of the sample at 950$1100{ }^{\circ} \mathrm{C}$, separation of the pyrolysis products $\left(\mathrm{CO}_{2}, \mathrm{H}_{2} \mathrm{O}\right.$ and $\left.\mathrm{N}_{2}\right)$ and their detection. Oxygen was determined by the difference between the mass of the sample and the total mass of all other elements: $\mathrm{O}=100 \%-\mathrm{X}(\% \mathrm{C}, \% \mathrm{H}, \% \mathrm{~N}$, $\ldots, \%)$. The degree of internal oxidation $(\omega)$ was calculated using formula $\omega=(2 \mathrm{O}-\mathrm{H}) / \mathrm{C}$.

The absorbance of humic acids was measured over the wavelength range 200$700 \mathrm{~nm}$ on a Lambda 35 Perkin Elmer UVvisible spectrophotometer.

Fourier transform infrared spectroscopy was recorded on a Perkin-Elmer spectrometer (SpectrumGX) over a scan range $4000-500 \mathrm{~cm}^{-1}$ using the $\mathrm{KBr}$ pellet method.

Scanning electron microscopy (SEM) was carried out on a SEM-100 microscope.

Total acidity was determined by the $\mathrm{Ba}(\mathrm{OH})_{2}$ method, and values for carboxylic groups were obtained through the Ca-acetate procedure [15]. Phenolic groups were calculated by subtracting carboxylic group from total acidity.

Reducing capacities of humic-like acids were determined using ferricyanide as oxidizing agent [16].

The ESR experiments were carried out with an E-500 Ecexys spectrometer (9.39231 $\mathrm{GHz}$ ), with a frequency modulation of $100 \mathrm{kHz}$. The solid samples were placed in a $2 \mathrm{~mm}$ diameter quartz tube. Magnetic field was $340 \mathrm{mT}$ and microwave power was $0.2 \mathrm{~mW}$ with a modulation amplitude of $0.2 \mathrm{mT}$ at room temperature. The concentration of paramagnetic centers was calculated from the known concentration of spins of coal pitch, which was $3.66 \cdot 10^{15} \mathrm{spin} / \mathrm{g}$.

\section{Synthesis of humic-like acids}

A portion of 1,2-naphthoquinone weighing $0.158 \mathrm{~g}$ was added to the Kjeldahl flask. After sealing the system, air was displaced from the flask with pure oxygen. Then, $2 \mathrm{ml}$ of a $1 \mathrm{M} \mathrm{NaOH}$ solution and $23 \mathrm{ml}$ of distilled water were added to the reaction flask. Mechanical shaking of the flask helped to mix thoroughly the contents of the flask.

During oxidation of 1,2-naphthoquinone with molecular oxygen under conditions of an excess of alkali, a mixture of synthetic humic substances is formed. During the synthesis, the solution acquired a dark brown color. The oxidation process was controlled volumetrically by measuring the amount of oxygen that was used during the reaction. Oxidation was carried out before to stop the marked consumption of oxygen. Under these conditions, the oxidation process lasted about 1 hour.

The humic-like acids were isolated from solution by precipitation at $\mathrm{pH}=2$ by the addition of $1 \mathrm{M} \mathrm{HCl}$. The resulting precipitate 
was filtered and washed three times with distilled water. The obtained synthetic humic acids were dried at $80^{\circ} \mathrm{C}$.

To study the kinetics of the oxidation reaction of naphthoquinone by molecular oxygen, a series of experiments was carried out with a molar ratio of naphthoquinone to alkali in the range from $1: 0.5$ to $1: 4$ (Fig. 2). The volume of oxygen used at each point in time was measured by a gas burette in which the blocking fluid was water. The molar ratio of used oxygen to 1,2-naphthoquinone $\mathrm{r}\left(\mathrm{O}_{2} / 1,2-\right.$ NQ) was calculated using the formula:

$$
\begin{aligned}
& r\left(\mathrm{O}_{2} / 1,2-\mathrm{NQ}\right)=\frac{n\left(\mathrm{O}_{2}\right)}{n(1,2-\mathrm{NQ})}= \\
& =\frac{\left(P_{a t m}-P_{\mathrm{H}_{2} \mathrm{O}}\right) \cdot V \cdot 273.15}{760 \cdot(T+273.15) \cdot V_{m}},
\end{aligned}
$$

where $r_{\mathrm{O}_{2}}-$ molar ratio $\mathrm{O}_{2}$ to naphthoquinone (NQ); $P_{\text {atm }}$ - atmospheric pressure, $\mathrm{mm} \mathrm{Hg}$;
$P_{\mathrm{H}_{2} \mathrm{O}}$ - water vapour pressure at the synthesis temperature, $\mathrm{mm} \mathrm{Hg} ; \quad V$ - volume of used oxygen, $\mathrm{ml} ; V_{m}$ - molar volume of gas under normal conditions, $\mathrm{mmol} / \mathrm{ml} ; T$ - temperature, $\mathrm{K}$.

The temperature dependence of water vapour pressure $(\mathrm{mmHg})$, can be represented by this equation:

$$
\begin{aligned}
& \mathrm{P}_{H_{2} O}=7 \cdot 10^{-6} \cdot t^{4}-3 \cdot 10^{-4} \cdot t^{3}+ \\
& +2.72 \cdot 10^{-2} \cdot t^{2}+0.1194 \cdot t+5.05
\end{aligned},
$$

where $t$ - temperature, ${ }^{\circ} \mathrm{C}$.

\section{Results and discussion}

When the ratio of naphthoquinone to alkali was more than $1: 2$, the time dependence of oxygen consumption during the process of oxidation reaction practically unchanged; therefore, this ratio is optimal for producing synthetic HAs (Figure 2, A).
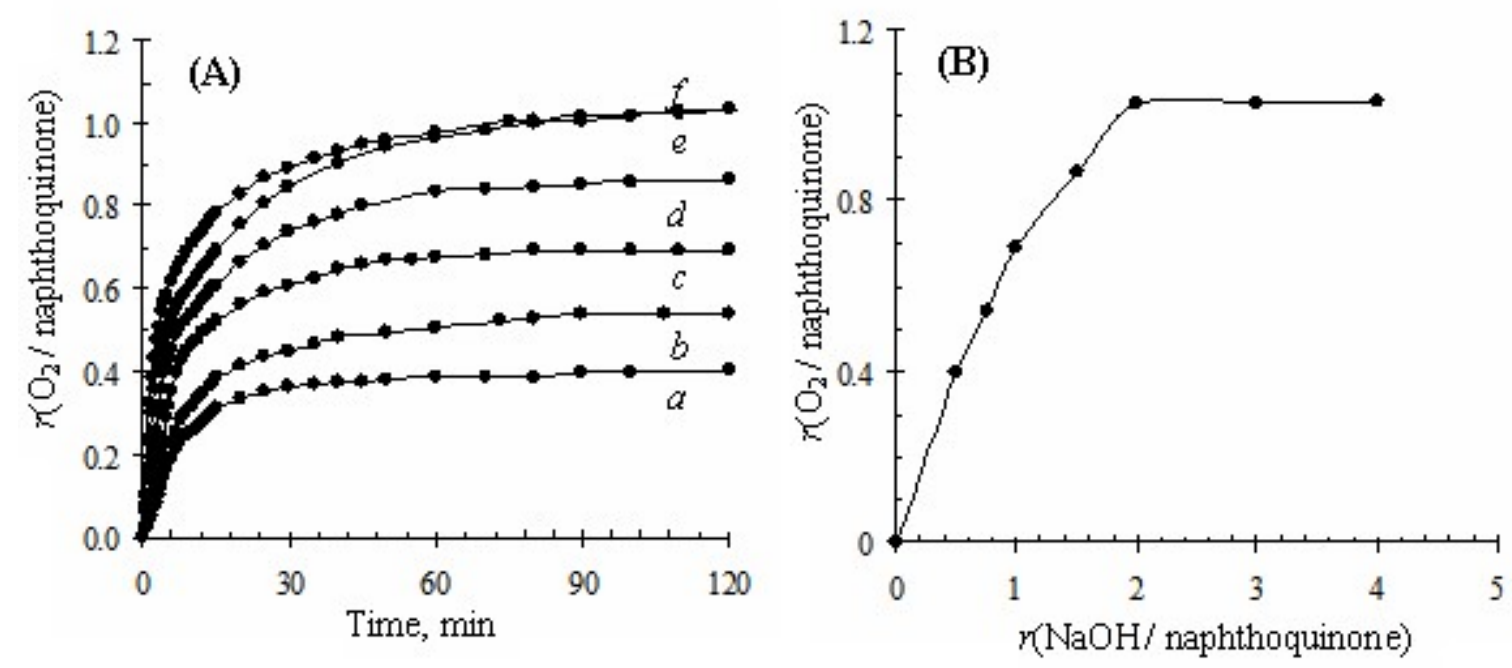

Figure 2. (A) Time dependences of the molar ratio $n(O 2): n(1,2$-naphthoquinone): 1:0.5 (a); 1:0.75 (b); 1:1 (c); $1: 1.5$ (d); $1: 2$ (e); 1:4 (f). (B) The molar ratio $\mathrm{n}(\mathrm{O} 2): \mathrm{n}(1,2$-naphthoquinone) versus the molar ratio $\mathrm{n}(\mathrm{NaOH}): \mathrm{n}(1,2-$ naphthoquinone) at $120 \mathrm{~min}$. 
The curve of the relationship in the absorption spectra, although their $\mathrm{n}(\mathrm{O} 2): \mathrm{n}(1,2-\mathrm{naphthoquinone})$ versus $\mathrm{n}(\mathrm{NaOH})$ : intensity decreased. With large amounts of $\mathrm{n}$ (1,2-naphthoquinone) allowed us to determine alkali used, maxima in the long wavelength the stoichiometry of the reaction, which is 1:2 region disappear, which indicates a deeper (Figure 2, B).

The UV-visible absorption spectra of 1,2-naphthoquinone and the reaction mixture with different ratios naphthoquinone to alkali are shown in Figure 3.

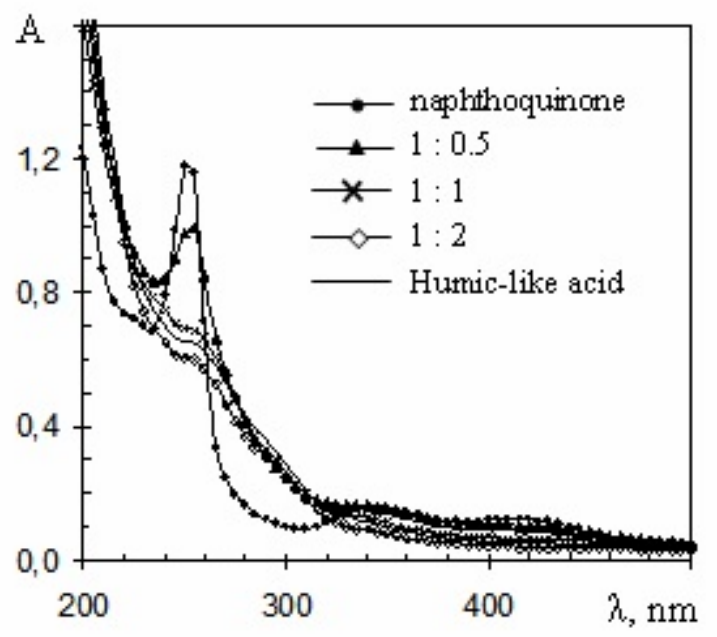

Figure 3. UV-Vis spectra of the 1,2-naphthoquinone, reaction mixture at different molar ratio 1,2naphthoquinone to alkali and final humic-like acid.

The absorption spectrum of the 1,2naphthoquinone has two weak $\pi, \pi^{*}$ bands at longer wavelengths $(340 \mathrm{~nm}, 420 \mathrm{~nm})$ and a strong $\pi, \pi^{*}$ band at shorter wavelengths (250 $\mathrm{nm}$ ). The absorbance at $260 \mathrm{~nm}$ corresponds to the presence of aromatic structures. The weak absorbances at $340 \mathrm{~nm}$ and $420 \mathrm{~nm}$ indicated the presence of $\mathrm{C}=\mathrm{O}$ functional groups. At low molar ratios of 1,2-naphthoquinone to alkali (less than 1:1), all three maxima that are characteristic of 1,2-naphthoquinone are present oxidation of the starting material and formation of humic-like substances. The electronic spectra of the final humic-like acids are characterized by continuous absorption in the shortwavelength region with a shoulder at $260 \mathrm{~nm}$. The absence of fine splitting in the spectra indicates that the contribution of the chromophore atomic groups to the total absorption is small. The polyconjugated system, due to the delocalization of electrons in molecular orbitals, causes an increase in the mutual influence of atoms and significant loss of individual bonds. The shoulder at $260 \mathrm{~nm}$ indicates the presence of aromatic fragments in the composition of humic-like acid and corresponds to known data, according to which the main chain of HA molecules was constituted from phenylcarboxylic acid fragments.

To confirm the similarity of the structure and properties of the obtained product with natural HA, a comprehensive study of the physicochemical properties of the obtained product was carried out.

The elemental composition of 1,2naphthoquinone and humic-like acid are shown in Table 1. All the obtained data were consistent with typical values for natural HA [3]. Low values of the $\mathrm{H} / \mathrm{C}$ ratio indicate a preponderance of aromatic components in the structure of HA. 
Table 1. Elemental analysis data for 1,2-naphthoquinone, synthetic humic acids and natural humic acid

\begin{tabular}{|l|c|c|c|c|c|c|}
\hline \multicolumn{1}{|c|}{ Substances } & $\mathrm{C} / \%$ & $\mathrm{O} / \%$ & $\mathrm{H} / \%$ & $\mathrm{H} / \mathrm{C}$ & $\mathrm{O} / \mathrm{C}$ & $\omega$ \\
\hline 1,2-naphthoquinone & 75.95 & 20.25 & 3.80 & 0.60 & 0.20 & -0.20 \\
\hline Humic-like acid & $56.1 \pm 0.2$ & $39.7 \pm 0.5$ & $4.2 \pm 0.1$ & 0.90 & 0.53 & 0.16 \\
\hline Natural HA [3] & $43-57$ & $35-47$ & $4-6$ & $0.85-1.28$ & $0.49-0.77$ & $0.16-0.54$ \\
\hline
\end{tabular}

The value of the $\mathrm{O} / \mathrm{C}$ atomic ratio depends on the degree of oxidation and increases with an increase in the number of hydroxyl, phenolic, and carboxyl groups. A comparison of this ratio for 1,2-naphthoquinone and synthetic humic acids indicates an increase in the content of oxidized fragments in the structure of synthetic macromolecules.

The parameter $\omega$ (the degree of internal oxidation) of the initial substance has a negative value, which indicates that it is not a highly reduced form. A positive value of $\omega$ for humic-like acids indicates a high degree of internal oxidation compared to the starting material, 1,2-naphthoquinone. The obtained value is in agreement with the data for natural humic acids [17].

Infrared absorption spectra confirm the similarity of the synthesized humic acids with the natural humic acids of various source given in the literature [3], which indicates an analogy of their structure (Figure 4).

The strong band in the region of 3300$3500 \mathrm{~cm}^{-1}$ with a maximum about $3420 \mathrm{~cm}^{-1}$ is due to the presence of $\mathrm{OH}$ groups bound by intermolecular hydrogen bonds. Absorption bands at 2920 and $2850 \mathrm{~cm}^{-1}$ are due to stretching vibrations of $\mathrm{CH}$ bonds in aliphatic $\mathrm{CH}_{3}$ and $\mathrm{CH}_{2}$ groups. In addition, asymmetric and symmetric deformation vibrations of the $\mathrm{C}$ $-\mathrm{H}$ bond in the $\mathrm{CH}_{2}$ and $\mathrm{CH}_{3}$ groups appear at $1375 \mathrm{~cm}^{-1}$. The band with a maximum at 1700 $\mathrm{cm}^{-1}$ belongs to the free carboxyl group $\mathrm{COOH}$. The presence of aromatic rings in HA molecules is indicated by the absorption band at $1620 \mathrm{~cm}^{-1}$, which is caused by stretching vibrations of conjugated double bonds of carbon atoms. A wide absorption band with a maximum at $1200 \mathrm{~cm}^{-1}$ is due to the hydroxyl group and the $=\mathrm{C}-\mathrm{O}-$ group. Symmetric stretching vibrations in the $\mathrm{C}-\mathrm{O}-\mathrm{C}$ groups are characterized by bands at about $1030 \mathrm{~cm}^{-1}$. Weak aromatic groups bands also appear at 740 and $580 \mathrm{~cm}^{-1}$.

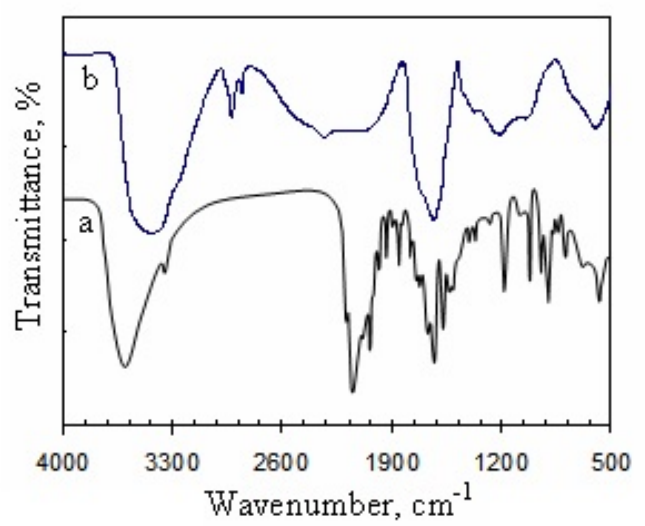

Figure 4. FTIR spectra of the 1,2-naphthoquinone (a) and humic-like acid (b). 
Table 2. Functional group contents of humic-like acids compared with natural HA

\begin{tabular}{|l|c|c|c|}
\hline Humic substances & $\begin{array}{c}\Sigma(\mathrm{ArOH}+\mathrm{COOH}) / \\
\mathrm{mmol} \cdot \mathrm{g}^{-1}\end{array}$ & $\begin{array}{c}-\mathrm{COOH} / \\
\mathrm{mmol} \cdot \mathrm{g}^{-1}\end{array}$ & $\begin{array}{c}\mathrm{ArOH} / \\
\mathrm{mmol} \cdot \mathrm{g}^{-1}\end{array}$ \\
\hline Humic-like acid & $8.0 \pm 0.3$ & $2.5 \pm 0.2$ & $5.5 \pm 0.2$ \\
\hline Natural HA [3] & $5.6-8.9$ & $1.5-5.7$ & $2.1-5.7$ \\
\hline
\end{tabular}

FTIR results support the formation of humic-like substances from oxidation of 1,2naphtochinon by molecular oxygen in alkali medium. All the absorption peaks observed in the humic-like acid spectrum have their counterparts in the spectrum of the natural humic substances reported in literature [3].

The content of functional groups is an important characteristic because they determine the reactivity of humic substances. Many characteristics of HA depend on the content of acid groups, such as polyelectrolyte properties, conformational variability and reactivity. The results of the content of oxygen-containing carboxyl and phenolic groups in the structure of synthetic humic acids (Table 2) are consistent with the literature for natural humic substances [3] and characterized by reproducibility.

For the characterization of free-radical species in humic-like acids electron spin resonance (ESR) spectroscopy was used (Figure 5). Humic-like acids give an ESR singlet signal with a width of 3.8 Gauss and $\mathrm{g}$-factor close to the g-factor of a free electron $(\mathrm{g}=2.0033)$. The presence of narrow ESR signals indicates that the humic-like acids are polymers with a well-developed system of conjugated bonds. The concentration of paramagnetic centers in humic-like acid is $15.0 \cdot 10^{16} \mathrm{spin} \cdot \mathrm{g}^{-1}$, which is in the range characteristic of natural HA extracted from various natural sources $\left(0.1-80 \cdot 10^{16} \mathrm{spin} \cdot \mathrm{g}^{-1}\right)$ [18].

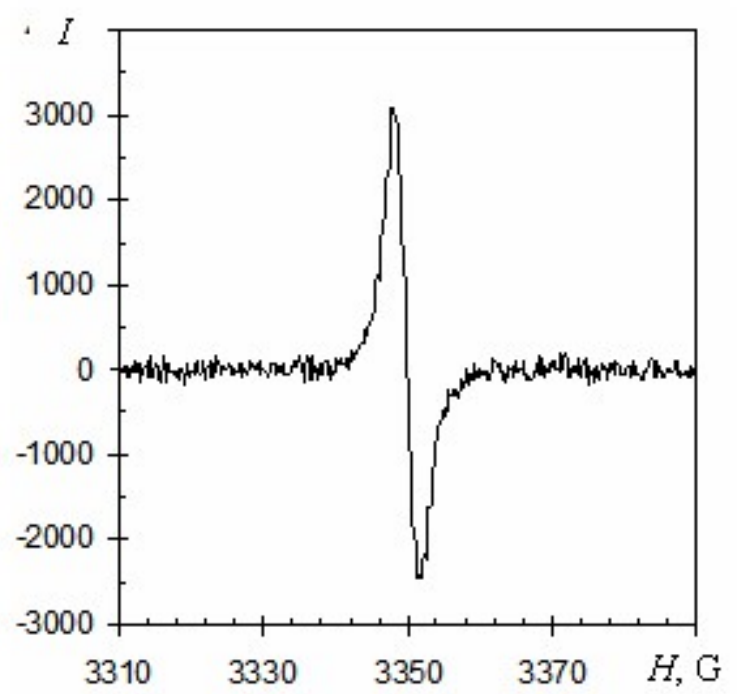

Figure 5. ESR spectrum of humic-like acid synthesized from 1,2-naphthoquinone.

Redox capacity was used for the quantitative evaluation of the redox properties of the obtained humic-like acid. Redox capacity is the amount of oxidant that is reduced by the interaction with synthetic humic substances normalized on their mass. The determination of this characteristic is 
Table 3. Redox capacity of humic acids at different $\mathrm{pH}$ values

\begin{tabular}{|l|c|c|c|c|c|}
\hline \multirow{2}{*}{} & \multicolumn{5}{|c|}{ Redox capacity, mmol·g ${ }^{-1}$} \\
\cline { 2 - 6 } & $\mathrm{pH}=5$ & $\mathrm{pH}=7$ & $\mathrm{pH}=9$ & $\mathrm{pH}=10$ & $\mathrm{pH}=11$ \\
\hline Humic-like acid & 0.43 & 1.45 & 2.41 & 2.99 & 3.68 \\
\hline
\end{tabular}

based on the reduction reaction of $\mathrm{Fe}$ (III) to $\mathrm{Fe}(\mathrm{II})$, which was carried out by reducing $\mathrm{K}_{3} \mathrm{Fe}(\mathrm{CN})_{6}$ to $\mathrm{K}_{4} \mathrm{Fe}(\mathrm{CN})_{6}$ with humic-like acids. The redox capacity was determined at different $\mathrm{pH}$ values. The obtained data are shown in Table 3. An increase of the redox capacity of synthetic humic substances with an increase in $\mathrm{pH}$ was found. A similar dependence was observed for natural humic substances [19]. Such a change in the redox ability with increasing $\mathrm{pH}$ is due to the different number of functional groups of humic-like acid that participate in the reduction process.

At high $\mathrm{pH}$ values, the humic acid have a conformation in which most of the functional groups (hydroquinone, ionized phenol, and carboxyl) are available for reduction. By reducing the $\mathrm{pH}$ of the humic acids solution, a compact spherical structure is formed due to intramolecular and intermolecular aggregation. In this case, access to the reduction of some groups in the humic acid structure is difficult because they are protected in the core [20].

The surface morphology of humic-like acid was studied by scanning electron microscopy. From SEM microphotograph, it results that humic-like acid form large associates with a smooth surface (Figure 6).

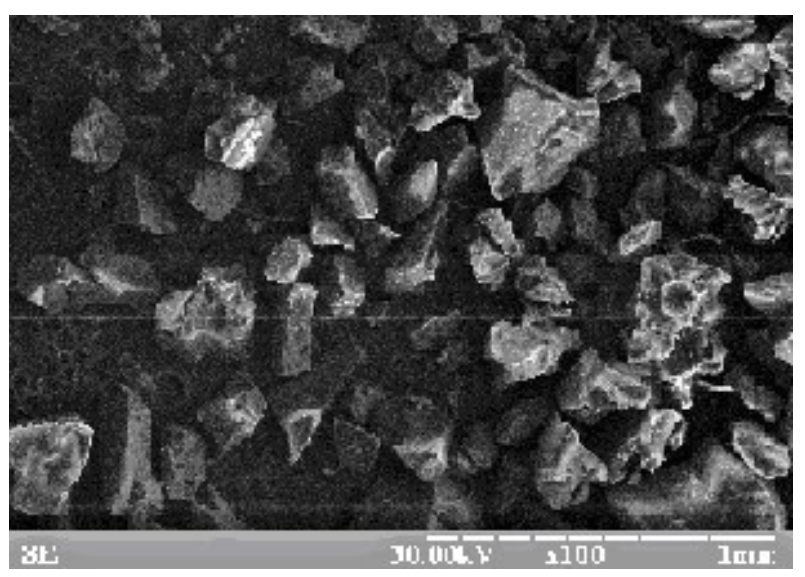

Figure 6. SEM image of the synthetic humic-like acid.

\section{Conclusions}

Specific organic substances obtained by the oxidation of 1,2-naphthoquinone with oxygen in an alkaline environment, based on the totality of the results of elemental, spectral and functional analysis, belong to the group of humic-like substances. A volumetric study of the oxidation process at various molar ratios of 1,2-naphthoquinone and alkali made it possible to establish the stoichiometry of the reaction and the optimal conditions for the synthesis of humic-like acids. Strict control of the synthesis conditions allowed to obtain a product with reproducible and standardized properties while preserving the main characteristic features of this class of 
substances. This advantage will significantly expand the areas of possible uses of synthetic analogs of natural humic acids, particularly in medicine.

\section{Acknowledgements}

This work was supported by the Ministry of Education and Science of Ukraine (Project No. 0120U100477).

\section{References}

[1] Stevenson FJ. Humus chemistry. Genesis, compositions, reactions. NY: Wiley and Sons, Inc; 1994, pp. 24-56.

[2] Jonesa MN, Bryan ND. Colloidal properties of humic substances. Adv. Colloid Interface Sci. 1998; 78: 1-48.

[3] Senesi N., Loffredo E. The Chemistry of Soil Organi Matter. In Soil Physical Chemistry, 2nd Ed. D.L.Sparks, Ed.), CRC Prss, Boca Raton, Ch. 6, 1999, pp. 239-370.

[4] Gomes de Melo BA, Motta FL, Santana MHA. Humic acids: Structural properties and multiple functionalities for novel technological developments. Mater. Sci. Eng. C 2016; 62: 967-974.

[5] Kala KJ, Prashob P KJ, Chandramohanakumar N. Humic substances as a potent biomaterials for therapeutic and drug delivery system-a review. Int. j. Appl. Pharm. 2019; 11: 1-4.

[6] Tomar NK, Yadav KP, Relan PS. Characterisation of humic and fulvic acids extracted with $\mathrm{NaOH}$ and $\mathrm{NaOH}-\mathrm{Na}$-pyrophosphate mixture from soils of arid and subhumid regions. Arid Soil Res. Rehabil. 1992; 6: 177-185.

[7] Jokic A, Wang MC, Liu C, Frenkel AI, Huang PM Integration of the polyphenol and Maillard reactions into a unified abiotic pathway for humification in nature: the role of $\delta$-MnO. Org. Geochem. 2004; 35: 747-762.

[8] Jung A, Frochot C, Villiéras F, Lartiges B,
Parant S. Interaction of pyrene flu-oroprobe with natural and synthetic humic substances: Examining the local molecular organiza-tion from photophysical and interfacial processes. Chemosphere 2010; 80: 228-234.

[9] Litvin VA, Minaev BF, Baryshnikov GV. Synthesis and properties of synthetic fulvic acid derived from hematoxylin. J. Mol. Struc. 2015; 1086: 25-33.

[10] Kiprop K, Coumon M, Pourtier E, Kimutai S, Kirui S. Synthesis of humic and fulvic acids and their characterization using optical spectroscopy (ATR-FTIR and UV-visible). Int. J. Appl. Sci. Technol. 2013; 3: 28-35.

[11] Mathur S, Schnitzer M. A chemical and spectroscopic characterisation of some synthetic analogues of humic acids. Soil Sci. Soc. Am. J. 1978; 42: 591-596.

[12] Litvin VA, Galagan RL, Minaev BF. Synthesis and properties of synthetic analogs of natural humic acids. Russ. J. Appl. Chem. 2012; 85: 296-302.

[13] Babula P, Adam V, Havel L, Kizek R. Noteworthy Secondary Metabolites Naphthoquinones their Occurrence, Pharmacological Properties and Analysis. Curr. Pharm. Anal. 2009; 5: 47-67.

[14] Pinho B, Sousa C, Oliveira J, Valentão P, Andrade P. Naphthoquinones biological activities and toxicological effects. In: Bitterlich A, Fischl S, editors. Bioactive compounds: Type, biological activities and health effects. New York: Nova Science Publishers; 2012. p. 181-218.

[15] Jeong CY, Park CW, Kim JG, Lim SK. Carboxylic content of humic acid determined by modeling, calcium acetate, and precipitation methods. Soil Sci. Soc. Am. J. 2007; 71: 86-94.

[16] Perminova IV, Kovalenko AN, SchmittKopplin P, Khatfield K, Hertkorn N, Belyaeva EY, Petrosyan VS. Design of Quinonoid-Enriched Humic Materials with Enhanced Redox Properties. Environ. Sci. Technol. 2005; 39: 8518-8524.

[17] Boguta P, Sokoowska Z. Statistical 
Relationship between Selected Physicochemical Properties of Peaty-Muck Soils and their Fraction of Humic Acids. Int. Agrophys 2014; 28: 269-278.

[18] Senesi N. Electron spin (or paramagnetic) resonance spectroscopy. In: Methods of Soil Analysis: Chemical Methods (D.L. Sparks, ed.), ASA-CSSASSSA Publ., Madison (USA), Ch. 11, 1996, pp.323356.

[19] Helburn RS, MacCarthy P. Determination of some redox properties of humic acid by alkaline ferricyanide titration. Anal. Chim. Acta 1994; 295: 263-272.

[20] Alvarez-Puebla RA, Garrido JJ. Effect of $\mathrm{pH}$ on the aggregation of a gray humic acid in colloidal and solid states. Chemosphere 2005; 59: 659-667. 